\title{
CONCEPTIONS OF EQUIVALENT IMPERFECTIONS IN ANALYSIS OF STEEL FRAMES
}

\author{
A. Machowski ${ }^{1}$ and I. Tylek ${ }^{2, *}$ \\ ${ }^{1}$ Assoc. Prof. D. Sc. Ph. D., Faculty of Civil Engineering, \\ Cracow University of Technology, ul. Warszawska 24, 31-155 Cracow, Poland \\ ${ }^{2}$ M. Sc, Faculty of Civil Engineering, \\ Cracow University of Technology, ul. Warszawska 24, 31-155 Cracow, Poland \\ *(Corresponding author: E-mail: ik2@poczta.fm)
}

Received: 17 October 2006; Revised: 2 May 2007; Accepted: 22 June 2007

\begin{abstract}
Bases of four different conceptions of the equivalent initial tilt utilized in elastic and post-elastic analyses of steel frames are under consideration. It turns out that conception and value of equivalent initial tilt depend on method of analysis and limit state conception. Reduction of initial tilt depending on number of columns per story is motivated if statistically based stationary gaussian random noise model of initial column tilt and bar-disk mechanical model of skeletal structure are applied.
\end{abstract}

Keywords: Steel frames, equivalent imperfection

\section{INTRODUCTION}

Formulas for initial frame tilt recommended by different normalization commissions and individual authors have similar multiplicative form

$\phi_{0}=\phi_{0}^{(1)} \cdot k_{c}(m) \cdot k_{s}(n)$.

However these formulas have very different coefficients: $k_{c}(m) \leq 1$ and $k_{s}(n) \leq 1$, depending on number of columns on story $m$ and number of stories $n$ applied for reduction of basic value of equivalent single story column tilt $\phi_{0}{ }^{(1)}$ (usually $\phi_{0}{ }^{(1)}=1 / 200$ ).

Application of the equivalent initial tilt in steel frames analysis for the first time has been recommended in ECCS [1] Specifications - without reduction (Figure 1) of basic value $\phi_{0}{ }^{(1)}$ $\left(k_{c}=k_{s}=1\right)$.

\begin{tabular}{|c|c|c|c|}
\hline No. & Source & $k_{c}(m)$ & $k_{s}(n)$ \\
\hline 1 & ECCS [1] & 1,0 & 1,00 \\
\hline 2 & ECCS [2] & $\frac{1}{2} \cdot\left(1+\frac{1}{m}\right)$ & 1,00 \\
\hline 3 & PKNMiJ [3] & $\frac{1}{2} \cdot\left(1+\frac{1}{\sqrt{m}}\right)$ & $\sqrt{\frac{5}{h}} \leq 1$ \\
\hline 4 & CEN [4] & $\sqrt{0,5+\frac{1}{m}} \leq 1$ & $\sqrt{0,2+\frac{1}{n}} \leq 1$ \\
\hline 5 & $\begin{array}{c}\text { Beaulieu \& } \\
\text { Adams [5] }\end{array}$ & $m^{-0.455}$ & 1,00 \\
\hline
\end{tabular}

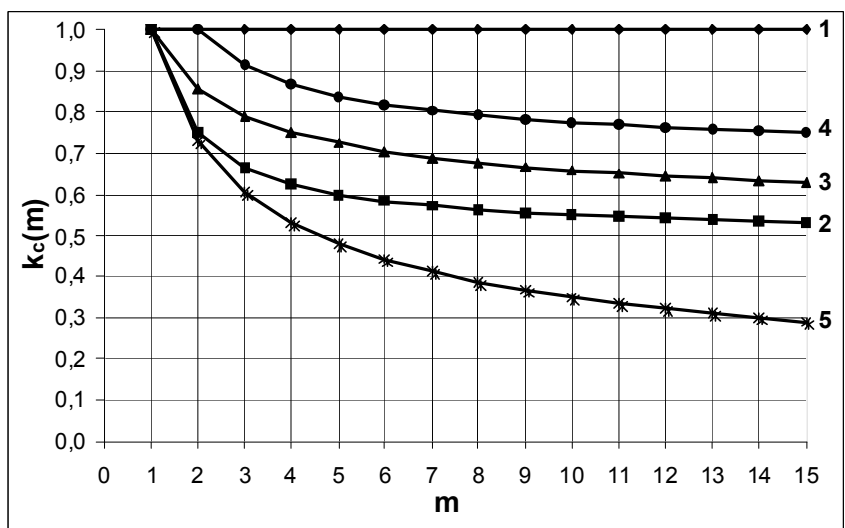

Figure 1. Comparison of Different Reduction Coefficients $k_{c}(m)$ and $k_{s}(n)$ or $k_{s}(h) ; h-$ Frame Height 
The smallest values of the reduction factor $k_{c}(m)$ have been proposed by Beaulieu and Adams (Beaulieu and Adams [5]), however they assume $k_{s}(n) \equiv 1$. In the paper mentioned above a letter $m$ denotes the number of columns on building story as a whole (not in single planar frame) - in contrast with other recommendations. Three factors in formula (1) are not yet satisfactorily justified. They are associated with steel frame erection tolerances and results of statistical analysis of post-erection column out-of-plumb measurements.

Applications of conclusions obtained from statistical analysis of column out-of-plumbs have been proposed among others by: Beaulieu and Adams (Beaulieu and Adams [5]) and Machowski (Machowski [6]). From theoretical analysis of the equivalent initial tilt problem utilizing statistically based initial column out-of-plumb model (Machowski [6], Machowski and Tylek [7]) follows that application of reduction coefficient $k_{s}(n)<1$ is not justified in general case of multistory frame analysis. However, applying other conceptions of equivalent initial tilt, some reduction coefficient $k_{s}(n)$ is justified, what is shown below.

\section{STATISTICALLY BASED INITIAL COLUMN TILT}

Column out-of-plumbs are related to orthogonal Cartesian coordinates system with axes parallel to structural system axes $(i, j, k)$ with $z$ axis oriented vertically and $x$ axis oriented along the building. With regards to statistical analysis it was more convenient to introduce initial tilt (interstory drift to story height ratio) as non-dimensional out-of-plumbness measure instead of horizontal deviations of column nodes. Definition of column $i$-th story initial tilts: $\phi_{i}^{x}$ and $\phi_{i}^{y}$ is illustrated in Figure 2.

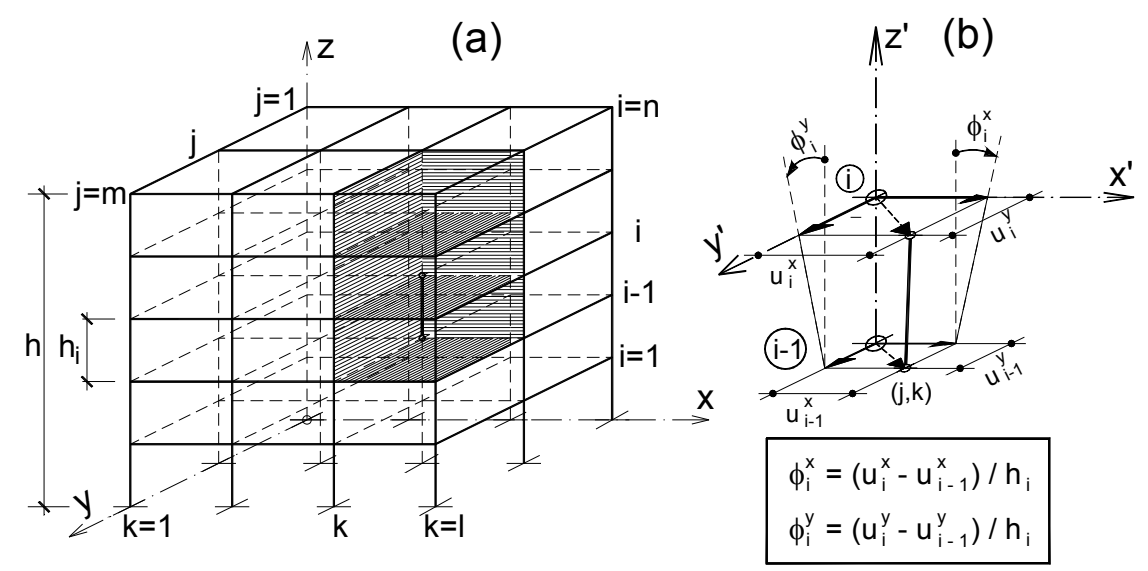

Figure 2. Elements of Columns Initial Tilt Matrix Definition

Horizontal deviations of column nodes from vertical lines, driven from central points of column bottom cross-sections: $u_{i}^{x}, u_{i}^{y}, u_{i-1}{ }^{x}, u_{i-1}{ }^{y}$ - respectively, are measured after all frame erection and after construction of all floors (post-fabrication measurements).

Statistical analysis of initial tilt measurements (Machowski [6], [8]) showed that mathematical model of stationary gaussian random noise $\phi_{i j k}{ }^{x}$ and $\phi_{i j k}{ }^{y}$ (stationary random series with mean value $\mathrm{E}\{\phi\}=0$ and constant variance $D_{\phi}=\sigma_{\phi}^{2}=$ const.) is justified if empirical variance fulfils a constraint

$\left.D_{\phi}=\sigma_{\phi}^{2} \leq 3(\%)\right)^{2}$ 
The second conclusion of this analysis was that exist only weak autocorrelation dependence between tilts of columns in the same vertical planar frames and lack of correlation between any other tilts. Proposed estimations of correlation coefficients extreme values are shown in Figure 3.

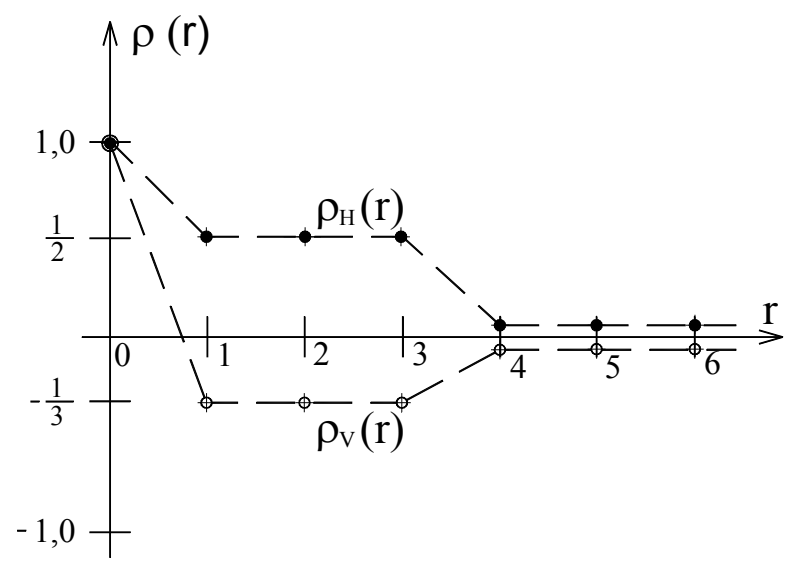

Figure 3. Assessment of Autocorrelation Dependences in Random Series of Initial Tilts $\left(\rho_{\mathrm{H}}(\mathrm{r})-\right.$ Autocorrelation in Horizontal Series, $\rho_{\mathrm{V}}(\mathrm{r})-$ Autocorrelation in Vertical Series $)$

For upper value of initial tilts according to Eq. 2 we obtain, in case of normal distribution, for allowable defectiveness $w=5 \%$ limit of tolerance applied in reception of structure

$\phi^{t}=1,96 \cdot \sigma_{\phi}=1,96 \cdot \sqrt{3} \%$ @ $1 / 295 \cong 1 / 300$.

The equivalent value of initial tilt from standard recommendation $\phi_{z}=1 / 200=5 \%$ has been justified in (Machowski [6]) as characteristic value of random variable which replace: initial column out-of-plumb, eccentricity in column splice and residual stresses. Respective value of variance $\left.\sigma_{\phi z}^{2}=(1 / 200 / 1,96)^{2} \cong(2,5 \%)^{2} \cong 6(\%)\right)^{2}$.

\section{EQUIVALENT INITIAL TILT IN ELASTIC analysis}

Determination of substitute random initial tilt for $i$-th story of building as a whole is the first step to determination of the equivalent initial tilt for all building.

Post erectional column out-of-plumbs cause additional bending moments $\underline{M}^{\phi x}$ and $\underline{M}^{\phi y}$ from vertical load for frame as vertical cantilever. A sequence of these cumulative moments $\underline{M}_{i}^{\phi}$ for $i$-th story and selective direction of initial tilt ( $x$ or $y$ ) may be written in following form

$\underline{M}_{i}^{\phi}=\underline{M}_{i+1}^{\phi}+\Delta \underline{M}_{i}^{\phi}$,

(random values are underlined)

For bar-disk analytical scheme (Machowski and Tylek [9]) of multistory skeletal structure (Figure 4) with rigid floor-disks effective initial random tilt for $i$-th story of building $\phi_{i}^{\text {eff }}$ may follow from conservation of global moment increment condition (if torsional effect for building as vertical cantilever is neglected) 


$$
\Delta \underline{M}_{i}^{\phi}=\sum_{p=1}^{s} P_{i p} \cdot h_{i} \cdot \underline{\phi}_{i p}=P_{i} \cdot h_{i} \cdot \underline{\phi}_{i}^{e f f},
$$

where: $P_{i}=\Sigma P_{i p},(p=1 \ldots s$.$) - sum of all vertical loads acting on building above i$-th story, $P_{i p}$ (for $s$ columns $p=1,2, \ldots, s$. on $i$-th story of building) - sum of vertical loads above $i$-th story in $p$-th column.

From Eq. 5

$\underline{\phi}_{i}^{e f f}=\sum_{p=1}^{s} w_{i p} \cdot \underline{\phi}_{i p}=\mathbf{w}_{i} \boldsymbol{\phi}_{i}^{\mathrm{T}}$.

Random tilt $\phi_{i}^{\text {eff }}$ is therefore a linear transformation of finite discrete gaussian noise $\phi_{i}=$ $\left[\phi_{i 1} \ldots \phi_{i p} \ldots \phi_{i s}\right]$ with deterministic weight-vector $\mathbf{w}_{i}=\left[w_{i 1} \ldots w_{i p} \ldots w_{i s}\right]$, where weights $w_{i p}=P_{i p} / P_{i}$ fulfil normalization condition $\Sigma w_{i p}=1,(p=1 \ldots s$.$) , is normal random variable with parameters:$

$$
\mathrm{E}\left\{\dot{\phi}_{i}^{e f f}\right\}=0, \quad \sigma_{\phi \mathrm{i}}^{2}=k_{c, i}^{2} \cdot \sigma_{\phi}^{2},
$$

where:

$$
k_{c, i}^{2}=\mathbf{w}_{i} \boldsymbol{\rho}_{s} \mathbf{w}_{i}^{\mathrm{T}}=\sum_{p=1}^{s} w_{i p} \cdot \sum_{q=1}^{s} w_{i q} \cdot \rho_{p q} \cdot
$$

A right side of expression Eq. 8 is positively defined quadratic form of $s$-variable: $w_{i 1}, \ldots, w_{i p}, \ldots w_{i s}$. with symmetric matrix of coefficients $\rho_{\mathrm{s}}$ equal to normalized correlation matrix $\rho_{\mathrm{s}}$ of random vector $\phi_{\mathrm{i}}$. Values of reduction coefficient $k_{c, i}$ according to Eq. 8 have been analysed in detail in (Machowski [6]) considering statistically based correlation matrix $\rho_{\mathrm{s}}$ and realistic range of weight-vector $\mathbf{w}_{\mathrm{i}}$ configurations.

Safe approximation of effective random initial tilt for frame as a whole may be defined as follows

$\underline{\phi}^{E F F}=\max _{i=1}^{n}\left|\underline{\phi}_{i}^{E F F}\right|$,

where:

$\underline{\phi}_{i}^{E F F}=\sum_{t=i}^{n} V_{t} \cdot \sum_{q=i}^{t} h_{q} \cdot \underline{\phi}_{q}^{e f f} \cdot\left(\sum_{t=i}^{n} V_{t} \cdot \sum_{q=i}^{t} h_{q}\right)^{-1}$

- effective random initial tilt for frame as a whole, which guarantee safe estimation of additional bending moment $\underline{M}_{i}^{\phi}$ for $i$-th story of frame as vertical cantilever, $\phi_{i}^{e f f}-$ according to Eq. 6 ,

$V_{t}-$ sum of vertical loads imposed immediately to $t$-th story.

The particular analysis of Eq. 9 and mentioned below numerical analysis has been made in (Machowski [6]). 
The numerically determined: probability density function $f(\xi)$ and distribution function $F(\xi)$, for effective frame initial tilt $\xi=\left|\Phi^{\mathrm{EFF}}\right|$, are showed in Figure 5.

The half-normal probability distribution of $\xi$ for $n=1$ is assumed (Machowski [6]). Top quantiles of $\xi$ (for example associated with value $\mathrm{F}(\xi)=0,95$ ) are almost independent on the number of stories (Figure 5). Therefore reduction of initial tilt standard deviation and characteristic value depending on story number $n$ is unfounded - in contrast to reduction depending on number of columns per story $m$.

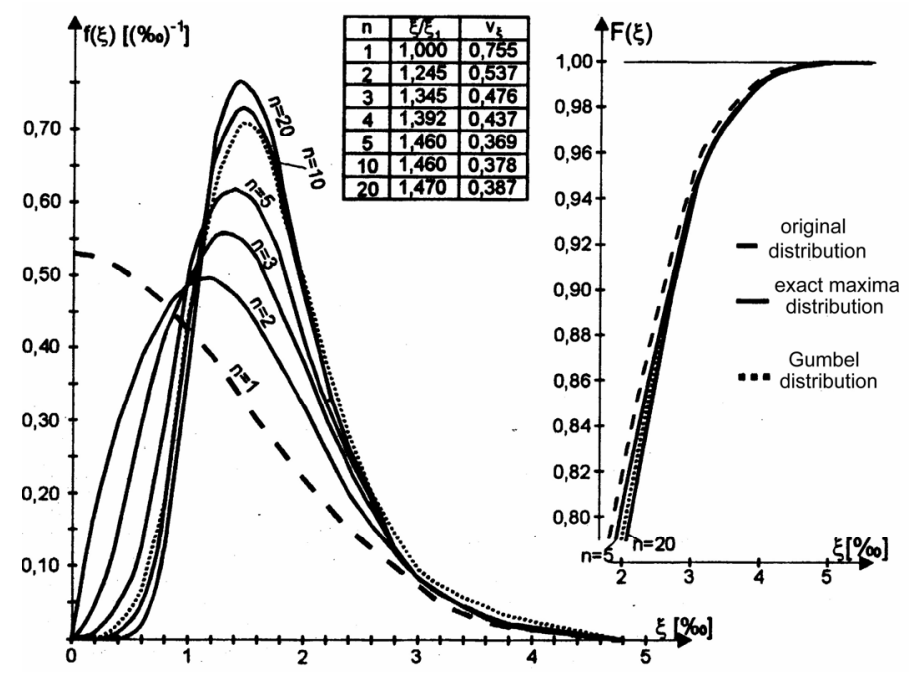

Figure 5. Comparison of Numerical Results for Equivalent Random initial Tilt of Frame as a Whole

$$
\xi=\left|\phi^{\mathrm{EFF}}\right|(n-\text { Number of Stories })
$$

Mentioned above conception of global effects of imperfection load conservation, independent on frame behaviour (elastic or post-elastic), may be replaced by conception of local effects of imperfection load conservation (i.e. in beam-column element of the skeleton). Using simplyfied linear beam-column resistance formula (Machowski [6])

$$
\Delta S=|\Delta N|+0,9 \cdot \varphi_{y} \cdot\left|\Delta M_{y}\right| / r_{y}=|\Delta N|+b \cdot\left|\Delta M_{y}\right|
$$

where: $r_{y}=W_{y} / A$.

The absolute value of equivalent initial tilt of frame as a whole is obtained from condition of local effect $\Delta S$ Eq. 11 conservation

$\underline{\phi}^{E F F} \cong\left(\left|\sum_{i=1}^{n} a_{N, i} \cdot \underline{\phi}_{i}^{e f f}\right|+b \cdot\left|\sum_{i=1}^{n} a_{M, i} \cdot \underline{\phi}_{i}^{e f f}\right|\right) /\left(\left|\sum_{i=1}^{n} a_{N, i}\right|+b \cdot\left|\sum_{i=1}^{n} a_{M, i}\right|\right)$,

where: $a_{N, i} ; a_{M, i}$ - coefficients of equivalent initial tilt of $i$-th story $\phi_{i}^{e f f}$ influence on axial force and bending moment, adequately, in selected element of the frame.

This conception was analysed on the example of the 5-story building B1 (Figure 6).

Building B1 has been designed according to the Polish Standard (PKNMiJ [3]) considering Polish load conditions. In this building five transversal steel rigid frames are braced by three longitudinal 
two-bay rigid frames, building has rigid in own plane reinforced concrete floors and light-weight curtain walls.

Tilt in $x$ axis direction of longitudinal frame in axis C-C was under consideration.

Substituting to Eq. $12 a_{N, i}, a_{M, i}$ and $b$ for the exterior column of the first floor of analysed frame and neglecting values of $a_{N, i}$ much smaller than $b \cdot a_{M, i}$, coefficient for conversion of variance of equivalent initial tilt of $i$-th floor $\sigma^{2}\left\{\phi_{i}^{\text {eff }}\right\}$ on variance of equivalent initial tilt of whole building $\sigma^{2}\left\{\phi^{E F F}\right\}$ is obtained

$k_{s}^{2} \cong\left|\sum_{i=1}^{5} a_{M, i}^{2}\right| /\left|\sum_{i=1}^{5} a_{M, i}\right|^{2}$.

Taking into consideration negative autocorrelation of $\phi_{i}^{\text {eff }}$ (in the plumb-line), reduction coefficient $k_{s}$ in analysed case is, according to Eq. 13 , equal to 0,894 . If this autocorrelation is passed over reduction coefficient $k_{s}$ obtained a little larger value $\left(k_{s}=0,907\right)$.

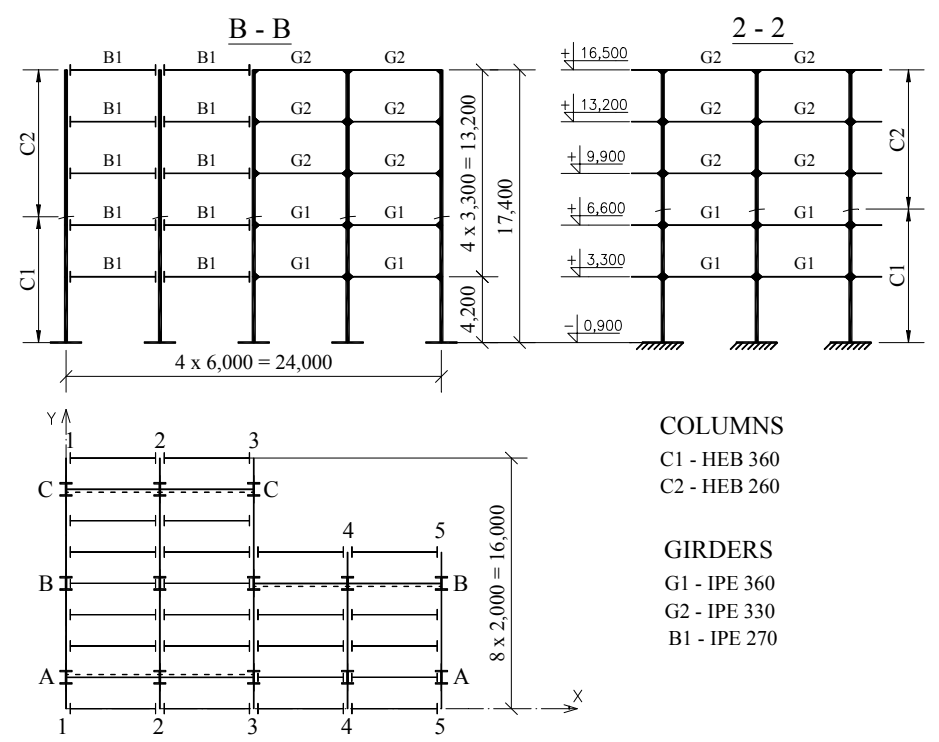

Figure 6. Analysed Skeletal Framed Steel Structure - Building B1 (5-Story)

\section{SUBSTITUTE INITIAL TILT IN POST-ELASTIC ANALYSIS}

Safe conception Eq. 9 of effective random initial tilt of frame, may be replaced by conditions of strength of frame (rigid-plastic or elastic-plastic) conservation.

To analyse conception of frame strength conservation four skeletal steel framed structures of multistory buildings were chosen (Figure $6-9$ ). Structures were analysed in post-elastic range with application of bar-disk analytical scheme (Machowski and Tylek [9]) neglecting torsional effects for building as vertical cantilever.

Tilt of transversal rigid frame in axis 4-4 is analysed in building B2 (Figure 7). This is the 9-story framed structure with 8 transversal carrying frames braced by 9 one-bay longitudinal rigid frames. 
16-story structure of building B3 (Figure 8) consist of 9 transversal carrying frames braced by 4 vertical trusses in longitudinal direction. Tilt of transversal frame in axis 5-5 is under consideration. Carrying system of 24-story building B-4 (Figure 9) has 4 transversal and 4 longitudinal steel rigid frames. Tilt of frame in axis 3-3 is analysed.

All skeletal structures under consideration have been designed according to the Polish Standard (PKNMiJ [3]) considering Polish load conditions. Buildings have rigid in own plane reinforced concrete floors and light-weight curtain walls.
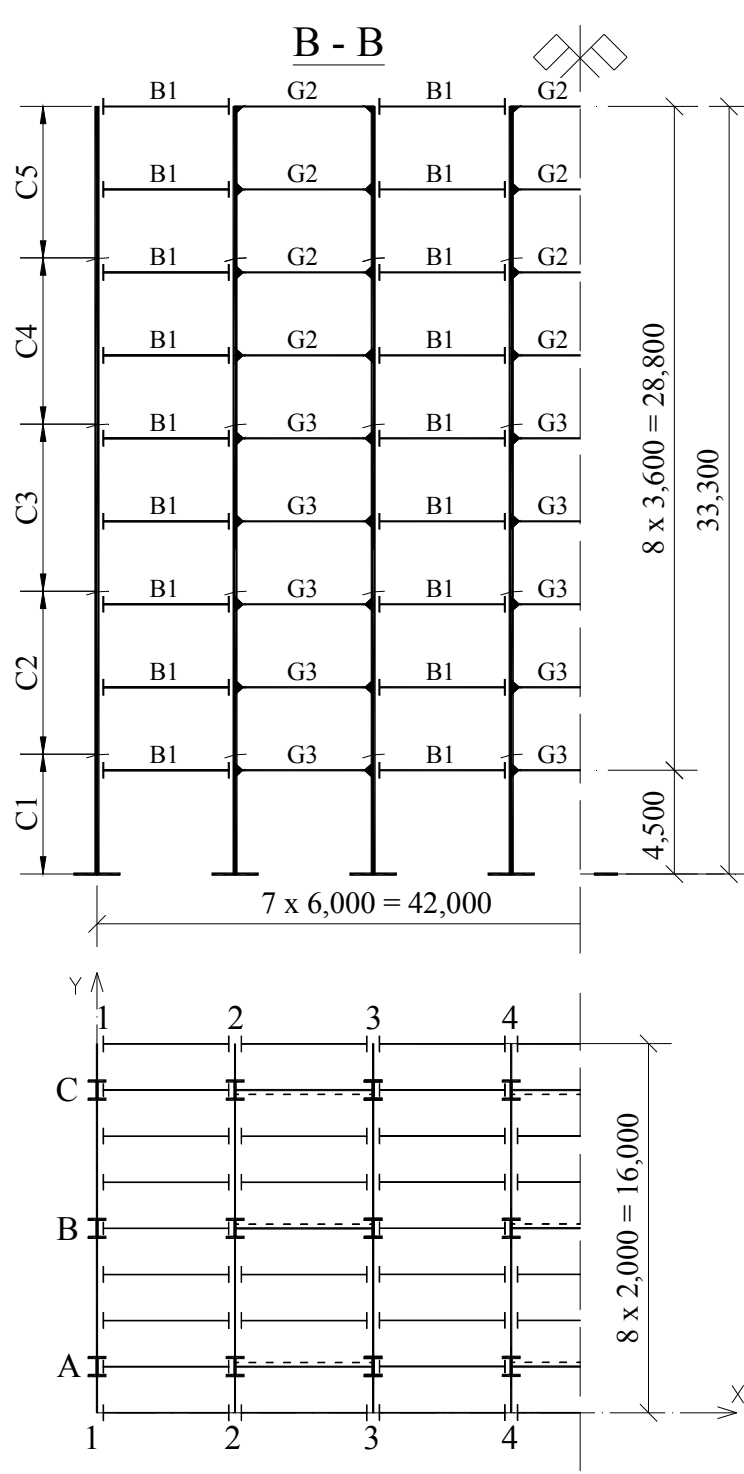

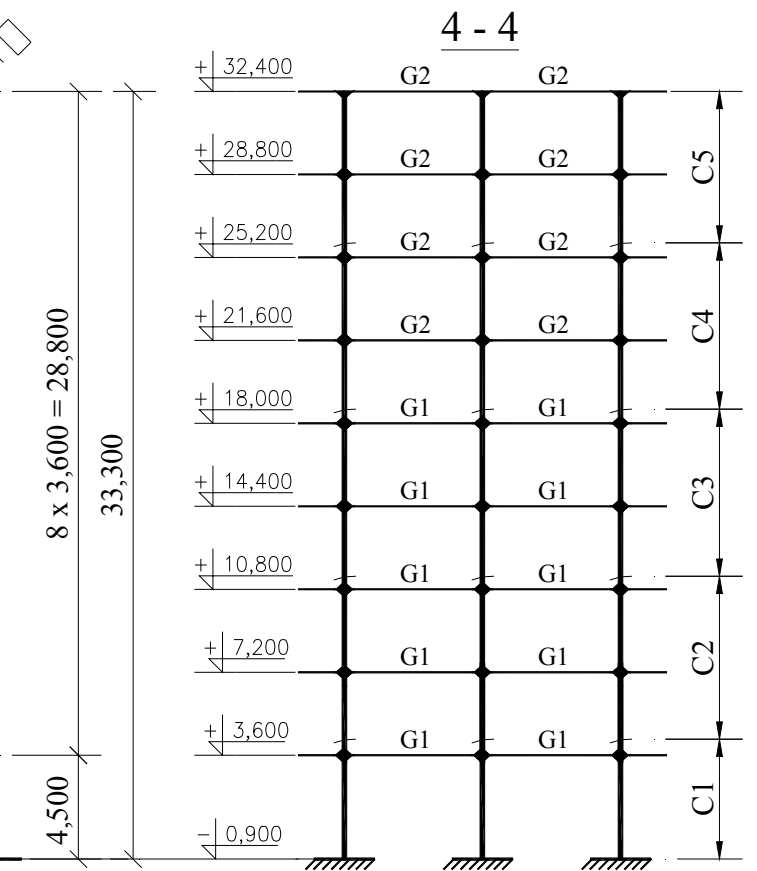

COLUMNS

C1 - I 510-15-510-30

C2 - I 480-13-480-28

C3 - I 480-13-480-30

C4 - I 460-13-460-28

C5 - I 440-12-440-25

GIRDERS

G1 - IPE 400

G2 - IPE 360

G3 - IPE 500

B1 - IPE 270

Figure 7. Analysed Skeletal Framed Steel Structure - Building B2 (9-Story) 


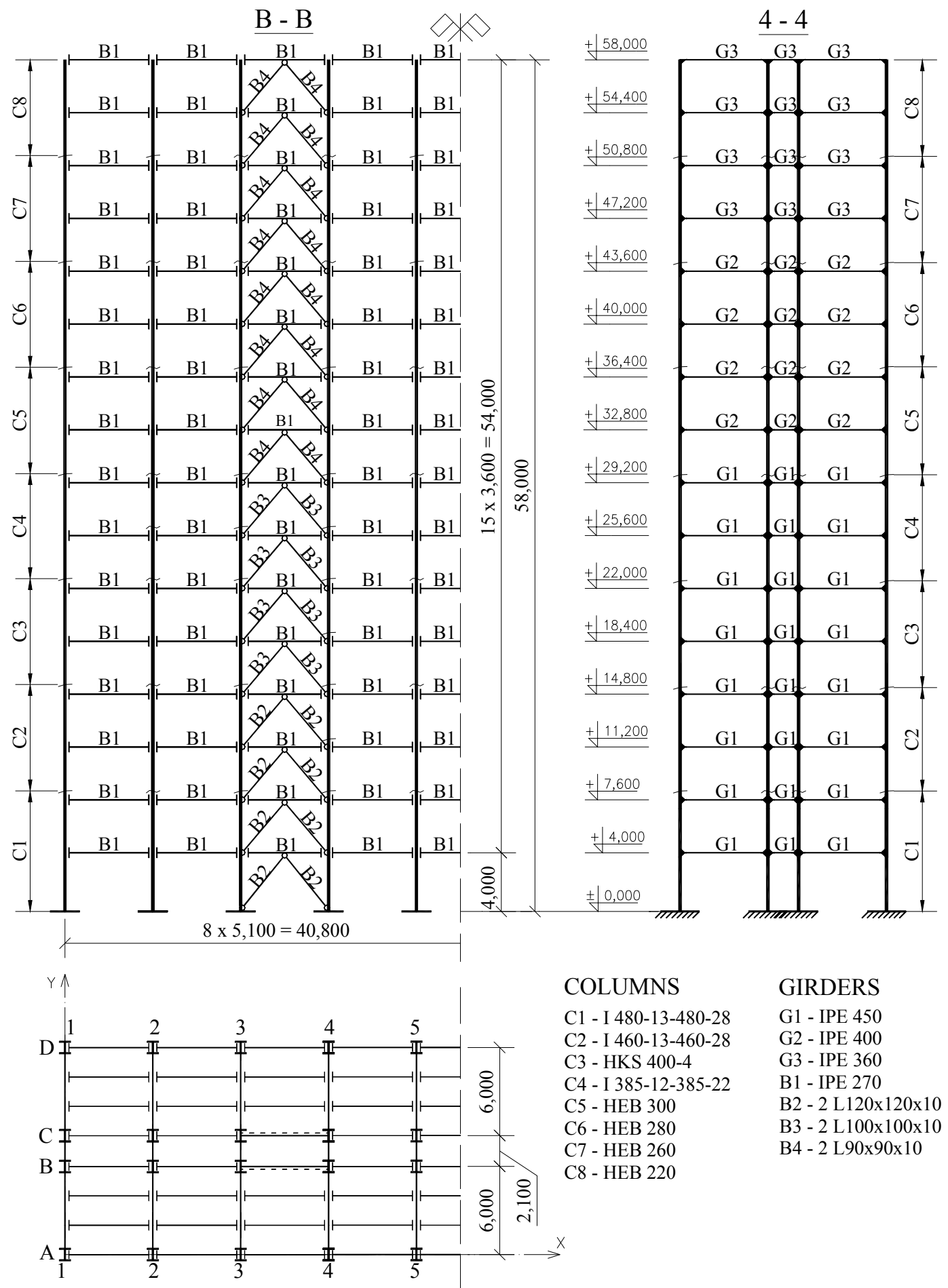

Figure 8. Analysed Skeletal Framed Steel Structure - Building B3 (16-Story) 


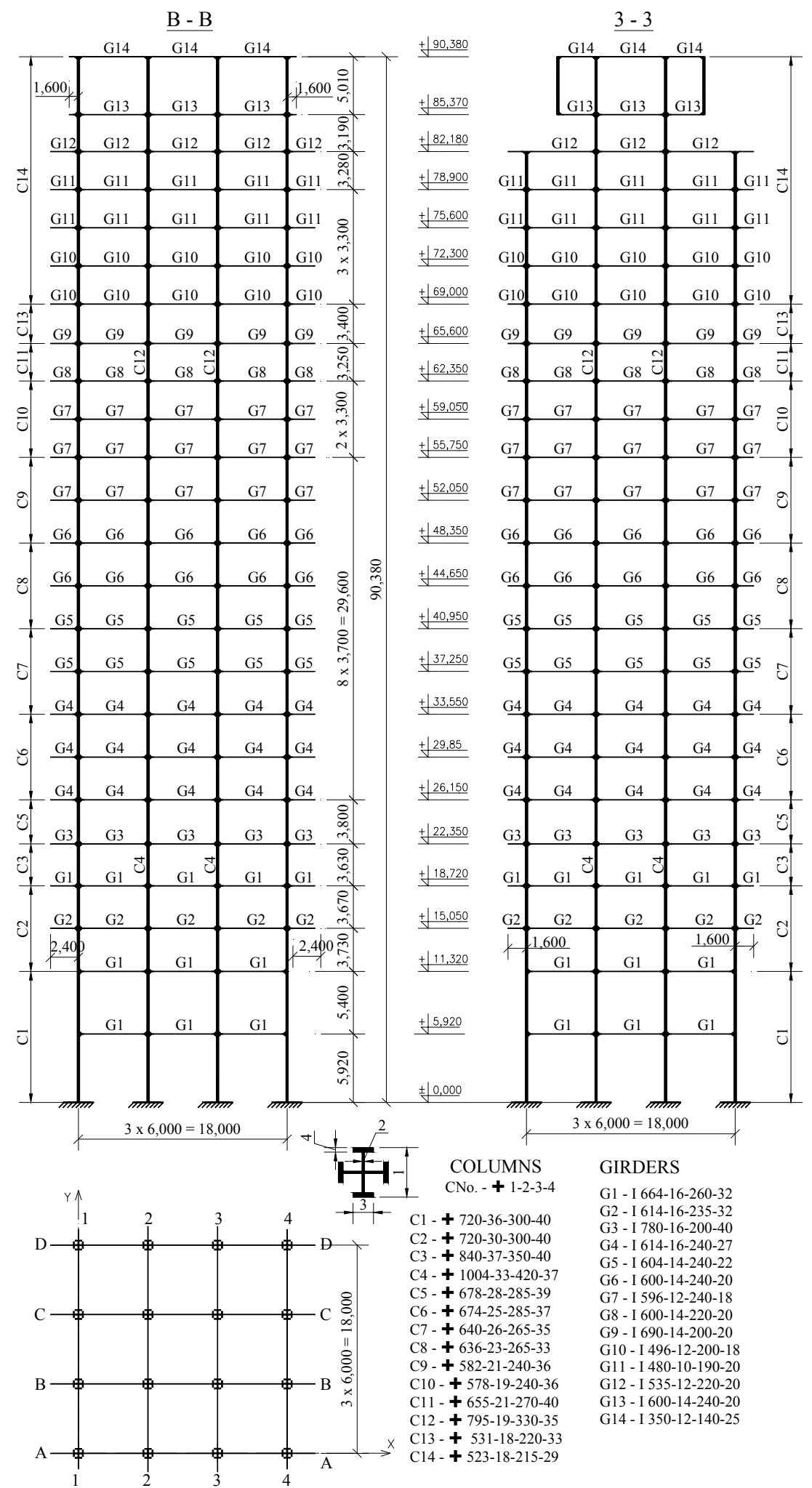

Figure 9. Analysed Skeletal Framed Steel Structure - Building B4 (24-Story)

In order to establish effective initial tilt two conceptions of frame strength conservation may be applied:

(A) conservation of power dissipated in plastic flow mechanism of complete solution and

(B) conservation of limit strength value associated with limit point $G$ on frame equilibrium path. 
According to ECCS recommendation (ECCS 1985) simple conception (A) is only valid if condition of "strong columns" (columns which remain elastic up to plastic mechanism formation) is fulfilled. Beams are designed in the first step (according to plastic mechanism) and next elastic columns are selected with application of equilibrium conditions.

Finally checking according to Merchant-Wood interaction formula is accomplished.

This design procedure is applied especially if seismic or other extraordinary loads are under consideration.

If random vector of initial effective story tilts $\left(\underline{\phi}_{I} \equiv \underline{\phi}_{i}^{\text {eff }}-\right.$ according to Eq. 6$)$ is replaced by a set of couples of forces $P_{i} \cdot \underline{\phi}_{i}$, according to Figure 10, then plastic mechanism with straight rigid columns and plastic hinges in beams may be under consideration. Conservation of dissipated power condition may be recorded in form (Figure 10)

$\dot{\omega} \cdot \sum_{i=1}^{n} P_{i} \cdot h_{i} \cdot \underline{\phi}_{i}=\dot{\omega} \cdot \underline{\phi}^{E F F} \cdot \sum_{i=1}^{n} P_{i} \cdot h_{i}$

where $P_{i}-$ as in Eq. 5,

from here effective initial tilt for frame as a whole may be expressed as linear transformation of vector of effective story tilts

$\underline{\phi}^{E F F}=\sum_{i=1}^{n} d_{i} \cdot \underline{\phi}_{i}$

where: $d_{i}=P_{i} \cdot h_{i} / \sum_{i=1}^{n} P_{i} \cdot h_{i}, \sum_{i=1}^{n} d_{i}=1$.

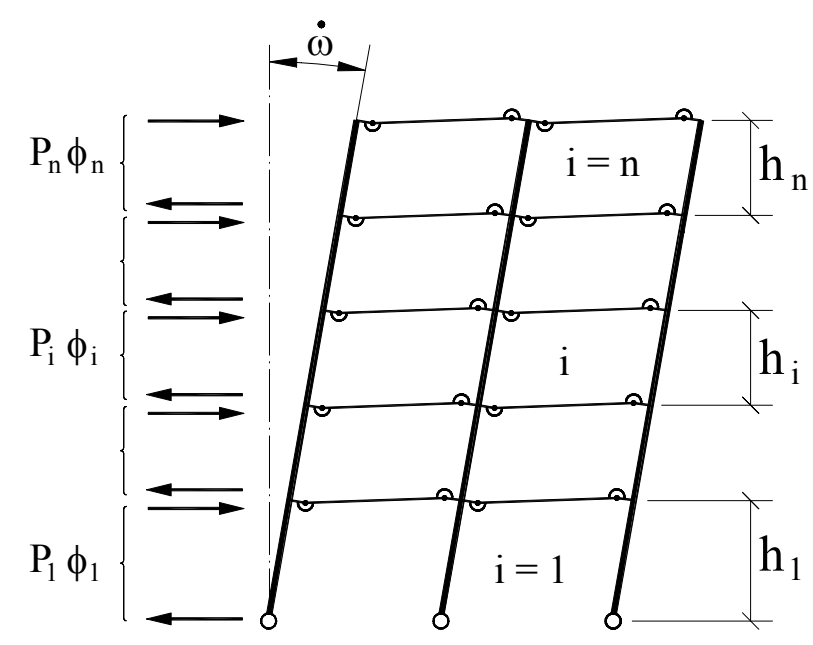

Figure 10. Collapse Mechanism of Frame with Strong (Rigid) Columns and Couples of Forces Replacing Random Initial Tilts of Stories

Reduction coefficients $k_{s}(n)=\sigma_{\phi E F F} / \sigma_{\phi i}$ - obtained according to Eq. 15 for buildings B1 - B4 are compared in Figure 13 with other results. 
Application of conception (B) (conservation of limit strength of frame value) to frames B1 - B4 requires appropriate computer application program. Program ANSYS has been utilized in order to obtain frame limit strength corresponding to limit point on equilibrium path (limit value of vertical load on frame $V_{R}$ corresponding to limit point on equilibrium path $G$ in Figure 11).

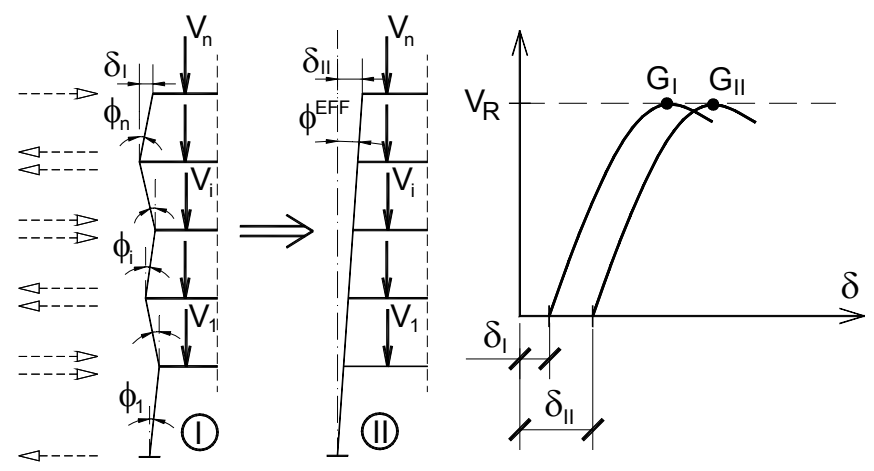

Figure 11. Sequence of Story Initial tilts, Effective Frame Initial Tilt $\phi^{E F F}$, Respective Couples of Forces and Equilibrium Paths

Elastic-plastic model of material with strain hardening coefficient $(1 / 10000) E(E=205 \mathrm{GPa})$ has been assumed. Post-limit (descending) part of equilibrium path has been obtained thanks to consideration of extended system of equations with nonlinear constraints equation (Crisfield's equation).

According to applied simulation procedure; for every realization of random effective story initial tilts sequence: $\phi_{1}, \ldots, \phi_{i, \ldots,} \phi_{n}$. respective frame equilibrium path (Figure. 11) has been determined. A concept of couples of forces replacing initial tilt of story is utilized. Next the effective initial tilt of frame as a whole $\phi^{E F F}$ has been chosen in agreement with condition $V_{R}\left(G_{I}\right)=V_{R}\left(G_{I I}\right)$ (Figure 11).

The results of calculations for building B2, as an example, are shown in Figure 12. According to results of statistical tests probability distribution of effective frame initial tilt $\phi^{E F F}$ may be approximated by normal distribution.

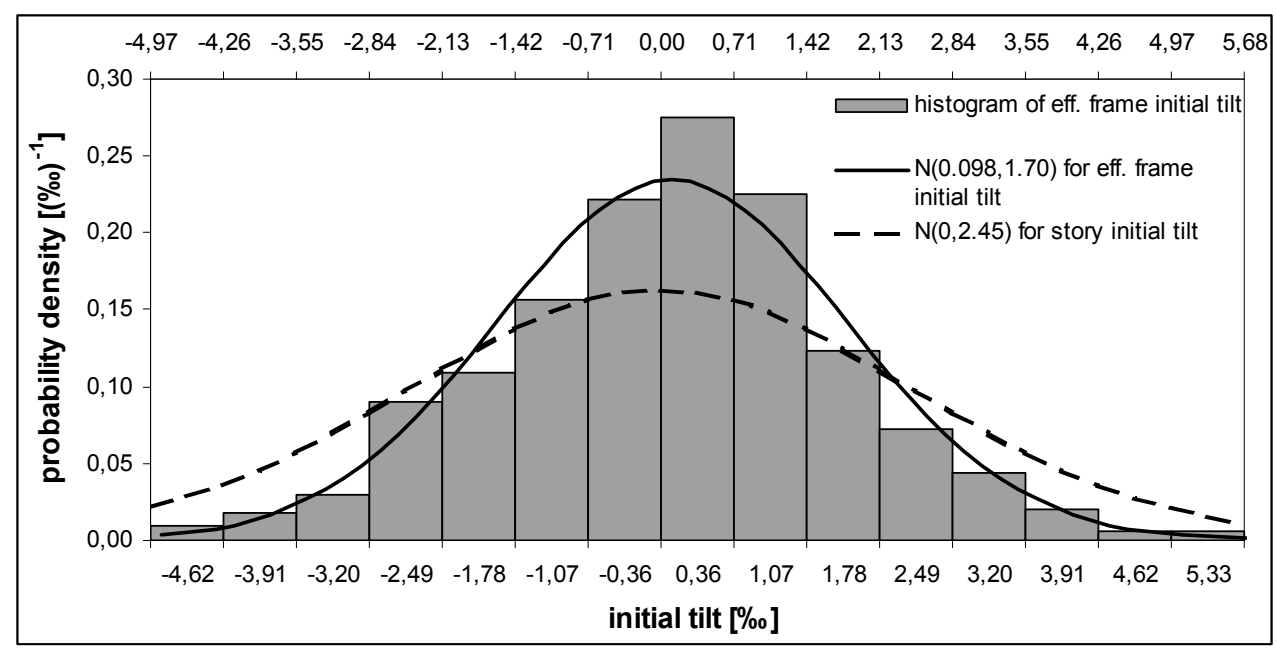

Figure 12. The example (building B2) of comparison story initial tilt and effective frame initial tilt $\phi^{E F F}$ probability distribution

Numerical results obtained for elastic and post-elastic analysis are compared with different codified recommendations for coefficient of reduction $k_{s}(n)$ in Figure 13. 


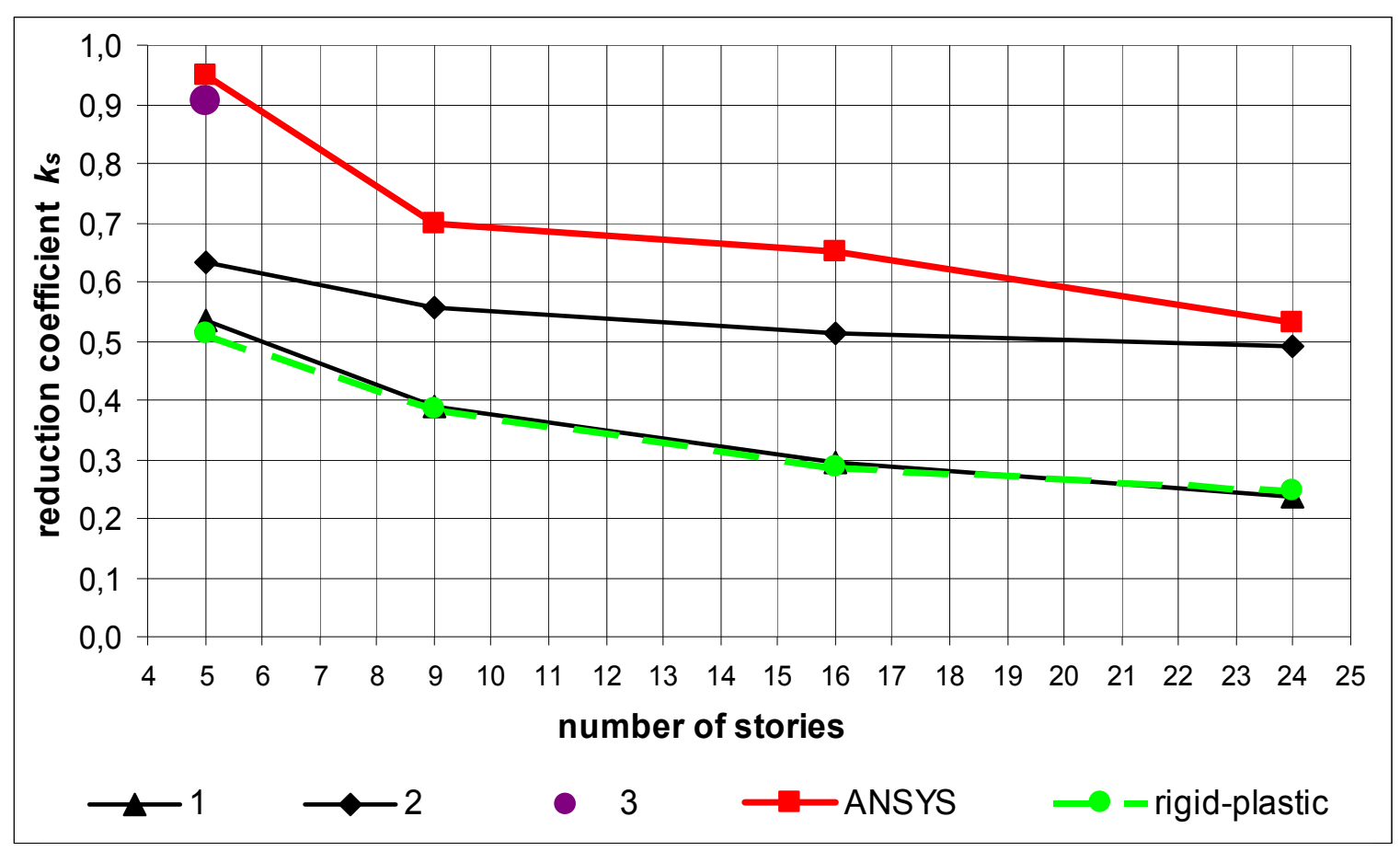

Figure 13. Comparison of Numerical Results and Reduction Coefficient $k_{s}(n)$ According to Different Suggestions (1: $k_{s}=\sqrt{5 / h}, h$ - height of the building, 2: $k_{s}=\sqrt{0,2+1 / n}, n-$ number of stories $, 3: k_{s}$ according to (I) conception)

\section{CONCLUDING REMARKS}

Main conclusion of conducted analysis may be formulated as follows: equivalent geometrical imperfection strongly depends on assumed ultimate limit state definition and method of analysis (elastic or post-elastic) so uniform formulae given in standards are questionable and are criticized in this article.

Reduction of the substitute initial tilt of steel frame depending on number of columns per story is sufficiently motivated if conception of bar-disk scheme and conservation of story moment conditions are accepted. This reduction may be applied in the same manner in elastic and post-elastic analysis.

A safe reduction of global imperfection depending on number of story is unjustified in general case. Some reduction of the equivalent initial tilt depending on number of stories is motivated if one of the following conceptions is applied: (I) imperfection load local effect conservation (in elastic analysis), conservation of limit strength (II) or dissipated power (III) (in post-elastic frame analysis). Values of reduction coefficient $k_{s}(n)$ determined for the same building according to these three conceptions differ from each other (i.e. for building B1 $k_{s}(n)=0,907$ according to $(\mathrm{I}), k_{s}(n)=$ 0,944 according to (II) and $k_{s}(n)=0,494$ according to (III)), what shows that standard recommendations for reduction factor $k_{s}(n)$ are unconservative in general case. It also indicate need of diversity of equivalent initial tilt value depending on type of analysis (elastic or elastic-plastic) and on conception of limit state (i.e. limit strength value associated with limit point on frame equilibrium path). 


\section{REFERENCES}

[1] ECCS 1974, European Specifications for Steel Structures, 1st Ed., 1974.

[2] ECCS 1984, Eurocode 3, Design of Steel Structures, 1984.

[3] PKNMiJ 1990, PN-90/B-03200, Steel Structures, Design Rules (in Polish), 1990.

[4] CEN 1990, CEN/TC 250, Eurocode 3: Design of Steel Structures, Part 1.1. General Rules and Rules for Buildings, 1990.

[5] Beaulieu, D. and Adams, P.F.,. "Significance of Structural Out-of-plumb Forces and Recommendations for Design", Canadian Journal of Civil Engineering, 1980, Vol. 7, pp.105-113.

[6] Machowski, A., "Problems of Limit States and Reliability of Steel Multistory Building Frames" (in Polish), Politechnika Krakowska, Monografia 262, Seria Inżynieria Lądowa, 1999.

[7] Machowski, A. and Tylek, I., "The Equivalent Imperfection Concepts of Steel Frame" (in Polish), L Konferencja KILiW PAN i KN PZiTB Krynica 2004a, Tom II, pp. 269-276.

[8] Machowski, A., "Initial Random Out-of-plumbs of Steel Frame Columns", Archives of Civil Engineering, 2002, XLVIII, Vol. 2, pp. 205-226.

[9] Machowski, A. and Tylek, I., "Application of bar-disk Systems in Analysis of Steel Frames" (in Polish), Czasopismo Techniczne, Politechnika Krakowska, 2004b, Budownictwo, Zeszyt Vol. 11, pp. 59-80. 